\title{
Fluoride Importance in Oral Manifestations
}

\author{
Medha Sansanwal $^{*}$, Shaifaly Chouhan ${ }^{2}$, Sumit Bhateja $^{3}$, Geetika Arora ${ }^{4}$ \\ ${ }^{\mathbf{1 - 2}}$ Dental surgeon, ${ }^{3} \mathrm{Head},{ }^{4}$ Reader, ${ }^{\mathbf{3}}$ Dept. of Oral Medicine \& Radiology, ${ }^{\mathbf{4}}$ Dept. of Public Health Dentistry, ${ }^{\mathbf{1 - 3}}$ Manav Rachna Dental \\ College, ${ }^{4}$ Inderprastha Dental College, Faridabad, India.
}

*Corresponding Author: Medha Sansanwal

Email: sansanwalmedha@gmail.com

\begin{abstract}
Fluoride is often called as nature's cavity fighter. It is a mineral that is present naturally in many foods and water. Whenever there is loss of mineral from the tooth enamel due to acids formed from plaque bacteria and sugars in the oral cavity the process is called as demineralisation. Fluoride, Calcium, and Phosphate are minerals which are redeposited to the enamel layer and this process is known as remineralisation. When there is excess of demineralisation without enough remineralisation to repair the tooth enamel it leads to tooth decay. Fluoride makes the tooth more resistant to acids formed from plaque bacteria and sugars. It reverses early signs of tooth decay. The fluoride taken in from foods and beverages provides a topical benefit as it becomes the part of saliva, constantly dipping the teeth with tiny amounts of fluoride that helps in rebuilding the weakened tooth enamel.
\end{abstract}

Keywords: Fluoride, Fluorosis, Fluoridation.

\section{Introduction}

Fluoride is the thirteenth most abundant element found in nature. It is present naturally in both water and air. Its concentration in water is liable to change. So, fluoride is added to public water supplies in order to bring the fluoride levels up to the mark necessary to help prevention of tooth decay. Other major sources of fluoride are seafood that contains edible bones or shells, tea, medicinal supplements, and fluoridated toothpastes. Stomach and small intestine absorbs the dietary fluoride rapidly. One-quarter to onethird of the absorbed fluoride is taken up into calcified tissues, whereas the rest is lost in the urine. In bone and teeth, fluoride can displace hydroxyl ions from hydroxyapatite to produce fluorapatite or fluorohydroxyapatite. ${ }^{1}$ Bones and teeth contain about $99 \%$ of the total body fluoride which increases steadily during life. For toddlers the adequate intake of fluoride id $0.7 \mathrm{mg}$ daily, which increases to $3 \mathrm{mg}$ daily for adult women and 4 mg daily for adult men. ${ }^{2}$ Fluoride has its beneficial effects like in bones, fluoride increases osteoblast activity and bone density, especially in lumbar spine. Low fluoride intake is only associated with the risk of dental caries, acting through both pre-eruptive and post-eruptive mechanisms.

Dental caries is a multifactorial microbial infectious disease adversely affecting most people in developing countries. Fluoride reduces the incidence of tooth decay and slows or reverses the progression of existing lesion. Although pit and fissure sealants, meticulous oral hygiene, and appropriate dietary practices contribute to caries prevention and control, the most effective and widely used approaches include fluoride use. Liquids and tablets of fluoride supplements are also available which should be prescribed by your dentist.

Water fluoridation is the "controlled adjustment of the concentration of fluoride in a communal water supply so as to achieve maximum caries reduction and a clinically insignificant level of fluorosis". The first 'artificial' water fluoridation for caries control was introduced in 1945 and
1946 in the United States (US) and Canada, respectively, and it was expected that caries prevalence would be reduced by as much as $50 \%$. As a result of the success of water fluoridation in controlling and preventing dental caries, several fluoride containing products like toothpaste, mouth rinses, dietary supplements, and professionally applied or prescribed gel, foam, or varnish were developed.

A major cause of fluorosis is the inappropriate use of fluoride containing dental products such as toothpaste and mouth rinses. The main aim of this review is to examine the importance of fluoride and its beneficial effects without being exposed to the adverse consequences of using it and to further examine the different aspects of fluoridation, their effectiveness in dental caries prevention and their risks.

\section{Mode of action ${ }^{3}$}

To control early dental caries, fluoride works in several ways. Fluoride has both topical and systemic modes of action. The fluoride interacts with the mineral component of the teeth and produces a mineral called fluorohydroxyapatite (FHAP or FAP), by substitution of $\mathrm{OH}$ - with F-. This results in an overall decrease in solubility and increased hydrogen bonding, a denser crystal lattice. The incorporation of fluoride into the hydroxyapatite (HAP) lattice may occur while the tooth is forming or by ion exchange after it has erupted. Greater amounts of fluoride incorporation increases the decrease in solubility, but rarely do we exceed several thousand parts per million of fluoride in the outer enamel. Therefore as compared to pure FAP that has 40,000 ppm fluoride, only limited protection from fluoride substitution could be expected. Another mode of incorporating fluoride into the enamel is from ion exchange and topical applications.

Following is the role of topical fluoride in prevention of dental caries:

1. Fluoride inhibits demineralisation - if fluoride is present in the plaque fluid at the time that the bacteria generate acid it will travel with the acid down into the subsurface 
of the tooth, adsorb to the crystal surface and protect it against being dissolved.

2. Enhances remineralization - Fluoride acts to speed up this remineralization process by adsorbing to the surface and acting to bring calcium and phosphate ions together, and is preferentially included in the chemical reaction that takes place, producing a lower solubility end-product inhibits plaque bacteria.

3. Interference with microorganisms - In high concentrations, fluoride is bactericidal and helps in reduction of plaque. Whereas in lower concentrations fluoride is bacteriostatic and helps controlling the growth of bacteria without destroying them. Fluoride lodges in plaque and inhibit bacterial enzymes responsible for acid metabolism.

4. Increased rate of posteruptive maturation - Fluoride increases the rate of mineralization of hypomineralized areas. Newly erupted teeth have hypomineralized areas which are prone to dental caries. Fluoride increases rate of mineralization and prevent from caries.

5. Modification in tooth morphology - Direct relationship exists between amount of fluoride ingested during tooth development and incidence of dental caries. If fluoride is ingested during tooth development the diameter and cusp depth of teeth are smaller. These changes decrease the caries susceptibility of teeth by making them more self cleansing.

6. After the eruption of teeth, fluoride works primarily especially when small amounts of fluoride are maintained constantly in the oral cavity, and specifically in saliva and dental plaque. Thus, not only children benefit from fluoride as was previously assumed, but also adults since it is more readily taken up by demineralized enamel than by sound enamel.

\section{Fluorosis $4,5,6$}

It is a chronic condition caused by excessive intake of fluorine compounds, marked by mottling of the teeth and, if severe, calcification of the ligaments.

\section{Source and Distribution of Fluorosis}

Sources of fluorosis are

1. Hydro fluorosis (water born)

2. Drug induced

3. Food born

4. Industrial dust \& fumes of fluoride

Distribution is as follow: About 25 countries all over the world are affected by fluorosis. In India at least 18 states are in alarming condition with fluorosis out of which 5 states are in severe endemic phase. Almost 50 to $100 \%$ districts of Tamil Nadu, Uttar Pradesh, Gujarat, Andhra Pradesh and Rajasthan are in endemic phase.

\section{Types of Fluorosis}

There are three types of fluorosis

1. Dental Fluorosis
2. Skeletal Fluorosis

3. Non Skeletal Fluorosis

\section{Dental Fluorosis}

Excessive intake of fluorine compounds leads to fluorosis a chronic condition marked by mottling of teeth. Dental fluorosis is hypo-mineralization of tooth enamel caused by intake of too much fluoride during enamel formation. It results in degrees of intrinsic tooth discoloration. The conditions severity is totally dependent on the dose, duration and age of the individual. ${ }^{7}$ In the mildest form (most common form) there are faint white lines or specks. Slightly more severe cases appear as white mottled patches, while severe fluorosis is characterized by brown discoloration and brittle, pitted and rough enamel.

In the extra-cellular environment of maturing enamel, an excess of fluoride ions changes the rate at which enamel matrix proteins (amelogenin) are enzymatically broken down and the rate at which the subsequent breakdown products are removed. Fluoride may also indirectly alter the action of protease via a decrease in the availability of free calcium ions in the mineralization environment. It leads to formation of enamel with less mineralization. Relative to normal enamel the hypo-mineralized enamel alters the optical properties and appears opaque and lusterless. Although dental fluorosis may be of potential cosmetic concern but are relatively resistant to dental caries. A number of other adverse effects have also been reported, viz., increased hepatic cell size, nephrosis, myocardial mineralization and degeneration of the seminiferous tubules in testis. The majority of fluoride is excreted via the kidneys therefore it is logical that those with impaired renal function might be at greater risk of fluoride toxicity.

\section{Water Fluoridation}

Water fluoridation is defined as "controlled adjustment of the concentration of fluoride in a communal water supply so as to achieve maximum caries reduction and a clinically insignificant level of fluorosis".

1. Optimum level of fluoride in water is approximately $1 \mathrm{ppm}$.

2. In water fluoridation, fluoride is ingested in public water supplies.

3. Water fluoridation is of two types, i.e. community water fluoridation and school water fluoridation.

\section{Community water fluoridation}

1. This is the controlled adjustment of fluoride in communal water supply so as to achieve maximum caries reduction and a clinically insignificant level of fluorosis.

2. Water fluoridation may be defined as an upward adjustment of the concentration of fluoride ion in public water supply.

3. The concentration of fluoride ion in the water may be consistently maintained at $1 \mathrm{ppm}$ by weight. 


\section{School water fluoridation}

1. By this the fluoride is available to children in optimal amount.

2. Amount of the fluoride which is added in the drinking water is more as compared to community water supply.

3. Currently recommended level of fluoride is $4.5-6.3 \mathrm{ppm}$ of fluoride in school water supply.

4. The concentration of fluoride in water is 4.5 times more, because the children remain in school for shorter period and also consumes less water.

5. School water fluoridation provides topical effects on erupting teeth.

6. It helps in prevention of dental caries in school children.

Water fluoridation helps in prevention of dental and root caries. It provides life-long benefits and also decreases the dental cost. Since the concentration of fluoride in drinking water is adjusted and continuously monitored, hence the adverse effects are avoided. It is the easiest method to reach more people at less cost and provide dental care but only less than $10 \%$ of the world's population has access to this intervention, as it is not feasible in many areas because of the nature of water supplies.

\section{Salt Fluoridation ${ }^{8,9}$}

Salt fluoridation was introduced by Wespi in year 1948 in Switzerland. It is a method for prevention of dental caries on mass level. Salt fluoridation consists of measured addition of fluoride at the time of manufacture of salt for community consumption. The level of fluoride in salt should be 200, 250 , and $350 \mathrm{mg}$ per $\mathrm{kg}$ of salt. The production of fluoridated salt takes place by two processes, i.e. batch processing and continuous processing:

1. Batch Processing - in this there is addition of fixed amount of fluoride in fixed amount of refined salt. For this process sodium fluoride is used. In this process most homogenous distribution of fluoride in a ton of salt is obtained after mixing it for 20 minutes.

2. Continuous Processing - this process takes place in large plants. In this process a dose of concentrated fluoride solution is sprayed via nozzle on the salt which is passing on a conveyor belt. Potassium fluoride is commonly used in this process.

Salt fluoridation has its own benefits like community water supply is not needed, individuals can accept or reject it, nonfluoridated salt as like noniodide salt can be available to the individuals who are in need. It also has some disadvantages like high salt intake can lead to hypertension, only refined salt can be used, and it requires a high technical expertise.

\section{Milk fluoridation ${ }^{10}$}

Milk fluoridation was introduced by Zeigler in Swiss city of Winterthur 1953. It consists of addition of measured quantity of fluoride to bottled or packed milk which was to be taken by children. For fluoridation of the milk sodium fluoride is added to the milk in form of concentrated aqueous solution in a fixed volume. If the requirement of fluoride is $1 \mathrm{mg}$ per day, concentration of fluoride in milk is set up at $5 \mathrm{ppm}$. Fluoridated milk should be distributed to children in kindergarten and nursery schools. The addition of fluoride to milk is a simple process and the costs of fluoridated and non-fluoridated milk are usually the same.

\section{Conclusion}

Fluoride is one of the most beneficial micro nutrients for human body but in excess can cause harm in many ways. According to recommendation of World Health Organization total daily fluoride exposure would be approximately $0.6 \mathrm{mg} /$ adult/day in an area in which no fluoride is added to the drinking-water and $2 \mathrm{mg}$ per adult per day in a fluoridated area. Although multifactorial in origin, caries is a preventable disease, with fluoride as a preventive agent used worldwide. To get the beneficial effects of fluoride, people must be careful before consuming fluoridated water, foods, salt and using highly fluoridated tooth paste. Several modes of fluoride use have evolved, each with its own recommended concentration, frequency of use, and dosage schedule. Government should monitor fluoride concentration in different sources of drinking water and ground water and thorough fluoride map should be available to the public.

\section{Source of Funding: None.}

\section{Conflict of Interest: None.}

\section{References}

1. Aoun A, Darwiche F, Hayek S, Doumit J. The Fluoride Debate: The Pros and Cons of Fluoridation. Prev Nutr Food Sci. 2018; 23(3).171-80.

2. National Research Council. Recommended dietary allowances. $10^{\text {th }}$ ed. The National Academies Press; Washington, DC, USA: 1989;235-40.

3. Wefel, J.S, Faller, R.V. A History and Update of Fluoride Dentifrices. dentalcare.com.

4. Dey, S. Giri, B. Fluoride fact on Human Health and Health problems. Medical and Clinical Reviews. December 24, 2015.

5. DunipaceAJ, WilsonCA, WilsonME, ZhangW, KafrawyAH. Fluoride in Drinking-water. World Health Organization (WHO). 2006.

6. Verma KS, What is Fluorosis its type, diagnosis and treatment. Indiastudychannel.com. 12 June 2011.

7. Bergc J.H, Slayton R.L, Early Childhood Oral Health. John Wiley and Sons. 2015. 113.

8. Marthaler TM. Salt fluoridation and oral health. Acta Med Acad. 2013;42:140-55.

9. Pollick HF. Salt fluoridation: a review. J Calif Dent Assoc 2013. 41. 395-7.400-04.

10. Banoczy J, Edgar M, Petersen PE, Rugg-Gunn AJ, Villa A, Woodward M. Milk fluoridation for the prevention of dental caries. WHO Press; Geneva, Switzerland 2009;156-67.

How to cite this article: Sansanwal $M$, Chouhan $S$, Bhateja S, Arora G. Fluoride Importance in Oral Manifestations. J Nutr Metab Health Sci 2019;2(4):97-9. 